\title{
The application of QoS technology based on NGN network
}

\author{
Jinyi Zhou \\ Sichuan Information Technology College, China \\ 30940350@qq.com
}

Keywords: NGN; Qos technology; Application.

\begin{abstract}
To analyze the implementation scheme of NGN in QoS, and conclude the key principles and requirements for implementation of NGN network QoS. To put forward a design scheme of NGN dynamic QoS based on mobile agent. And the related principle of the scheme, key points are introduced. The mobile agent technology which base on the distributed computing was realized QoS in NGN network. The QoS scheme can effectively reduce the amount of data acquisition, increased network traffic which caused by the QoS management information transmission. At the same time, it reduces the computational load of equipment management RCS. To help improve the response efficiency of QoS control system, reduce the burden of network transmission QoS management and control activities bring. In complex networks, it has excellent performance in large-scale network application.
\end{abstract}

\section{Introduction}

With the rapid development of IP technology and computer communication technology in the 90 years of the last century, people realized that the traditional telephone network based on circuit switch, computer network and cable TV network triple play will eventually converge to the unified exchange with IP based packet switching network. It is Next generation network NGN (Next Generation Network) ${ }^{[1]}$. IP technology makes all kinds of services which based on IP can interoperate in different online. The man has unified communication protocol which three networks can accept. Although the next generation network technology there are still different views and arguments, the soft switch (soft Switch) as the core of the NGN technology has gradually become the consensus of the industry. That is the development trend of next generation network. All major telecom operators have to carry out large-scale commercial deployment, a network communication technology revolution has been kicked off.

With the expansion of the NGN test and commercial network, to provide integrated services to NGN QoS (Quality of Service) will be more and more prominent ${ }^{[2]}$. NGN uses the IP technology to carry multiple services. But the traditional IP network can only provide "best effort" service. It far exceeded the traditional IP's ability on the requirements of audio, video and other real-time multimedia transmission. In this context, NGN provides telecom business based on the IP net, it has important theoretical value and practical significance to research NGN QoS.

\section{NGN and QoS Technology Overview}

\subsection{NGN}

Next generation network (NGN) is the core idea of business driven. It separates the business and transmission, transmission and control ${ }^{[3]}$. NGN does not require the data service, voice service, and the video service combination on the physical level. But in the network layer (IP layer), it achieves the integration. It provides system architecture and a unified open platform for voice, data, video and other kinds of business in the transmission of all kinds of physical network. "IP Over Everything, everything over IP" can achieve.

\subsection{QoS Technology}

The international standardization organization has a variety of equivalent or complementary definitions about QoS. The most famous of the ITU standard of QoS described as: QoS is a comprehensive reflection of a service performance. This determines the service performance to 
meet the business requirements of users in the network extent. QoS is a series of services to meet the requirements of data transmission in network flow requirements. The concrete can be quantified as the bandwidth, delay, delay jitter, packet loss rate, and throughput performance ${ }^{[4]}$.

\section{Study on Application of QoS Technology based on NGN}

\subsection{The principles and requirements of the realization scheme of QoS technology in NGN}

QoS problems of NGN business is the core problem of NGN business. As the mechanism and equipment of NGN QoS control, its performance has great influence on the application of NGN service. Determine the overall network QoS performance is mainly decided by the two levels NGN: bearer service layer and business layer. The relationship between two aspects of QoS parameter are interaction and mutual influence, instead of a single level decision QoS index to another level. The bearing layer of the QOS parameters such as delay, delay jitter, packet loss rate and bandwidth etc, it influenced the communication quality of the business layer, such as voice intelligibility and voice jitter. The service admission to the business control (Admission Contort) l would directly affect the overall network traffic. It effect of time delay, delay jitter and packet loss rate of network layer QoS index ${ }^{[5]}$. Therefore, the correct control strategy of NGN QoS network should follow the following principles:

The business layer of business admission control should based on the use of communication resources bearing layer situation;

Bearing control layer on the flow of business should be based on the business requirements of the transmission performance;

The business layer and the bearing layer should establish effective consultation mechanism, through information interaction between a feedback mechanism control two levels QoS.

As an effective QoS control scheme, in addition to the relevant principles of QoS meet outside, this scheme must carefully consider the computational efficiency of the problem, traffic problems, effective control, practical operational problems and high reliability.

\subsection{Design and research of the implementation of NGN dynamic QoS based on mobile agent}

\subsubsection{The design of NGN dynamic QoS scheme based on mobile agent}

The management response time and QoS management information flow of the traditional simple network management protocol SNMP are low. With the expansion of the network, its performance to a higher rate of linear upward trend. So it is not suitable for the fast developing trend under the complex network of information technology, QOS management of large networks. Therefore, the research and implementation of NGN network QoS. It presents a NGN dynamic QoS control scheme based on mobile agent. The control system model is shown in Figure 1. RCS (Resource Control Server) according to the different QoS tasks, it sent the corresponding mobile agent to each router or service control server to perform administrative tasks. The mobile agent returns RCS with the related management information after the completion.

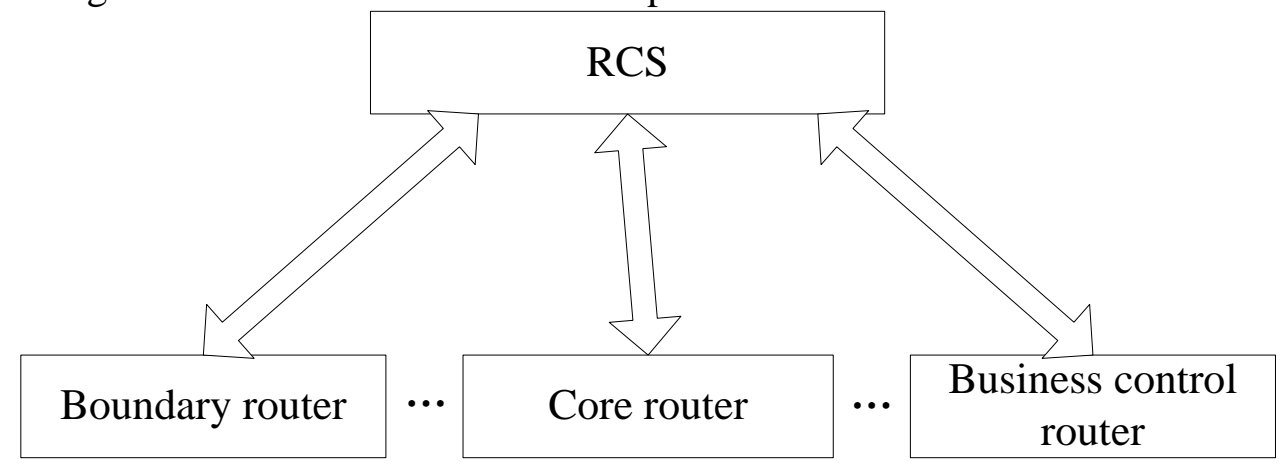

Figure 1. NGN dynamic QoS control system based on mobile agent

NGN dynamic QoS management model based on mobile agent, mobile agents create different functions according to the control requirements of the QoS. It transferred to each managed node (router and service control server) and control QoS flexible. It is mainly divided into two parts. One part is in RCS. Another part is on the management node router or service control server. As shown 
in Figure 2.

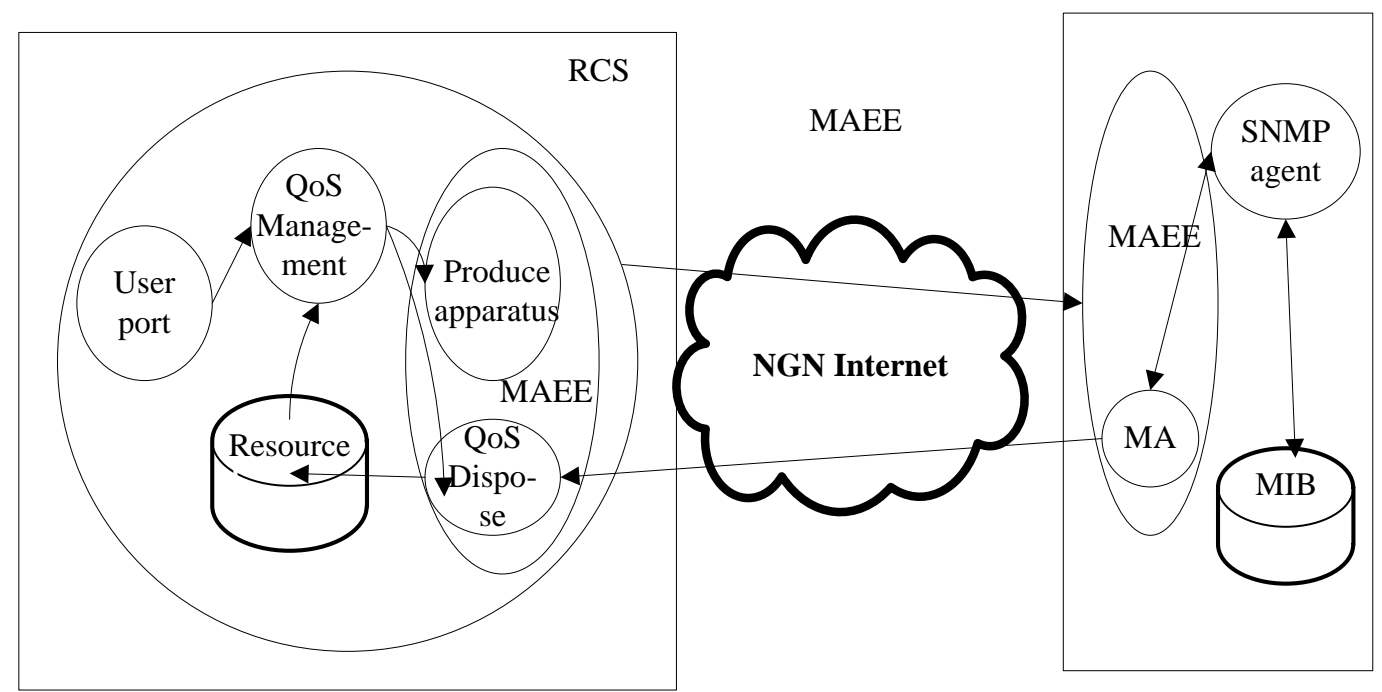

Figure 2. NGN dynamic QoS control system based on mobile agent

Rcs is responsible for coordinating the entire Qos dynamic control system operation. According to the certain Qos control task, it had a corresponding mobile agent. It sent to the managed node to perform QoS processing task. It also provides the user interface for the network administrator.

3.2.2 The advantages of NGN dynamic QoS scheme based on mobile agent

Based on the traditional SNMP scheme, it proposed scheme of dynamic QoS which based on mobile agent. In order to make up for the deficiency based on the scheme of SNMP.

Mobile agent is to avoid excessive management information transmission due to increased communication overhead solution framework. It moved request dynamic client code to the server without going through the intermediate link of network transmission. To interact with the service source directly. It improves the efficiency, reduces the branch network bandwidth requirements. Mobile agent can move freely between nodes in the network. It can carry their state and code in the network to migrate to another environment resume after created in an execution environment. Mobile agent has the mobility, autonomy, intelligent characteristic and strong ability of distributed computing. It can transfer to the local resources, to access resources and make the decision according to local mode. It is not required to send a permanent connection with the mobile agent. It avoids costly data transmission in congested networks. It responds for the change of the environment in real time. Obviously, the performance of mobile agent can effectively solve the computation efficiency of the NGN dynamic QoS implementation scheme of the key consideration, traffic control problem and high reliability issues.

\section{Conclusion}

This paper first introduces the NGN network architecture, the definition of QoS and study on the current situation of QoS network in NGN. According to the analysis of the implementation of NGN in QoS related features, put forward a design scheme of NGN dynamic QoS based on mobile agent. The relevant principles of the scheme, the key points are introduced. The comprehensive analysis shows that the mobile agent technology based on distributed computing. Through the "mobile computing" intelligence to equipment management, it reduces a large amount of data acquisition scheme based on QoS dynamic prediction, and network traffic increased caused by the QoS management information transmission. It improves the response efficiency of QoS dynamic control system, and reduce the burden of network transmission which caused by QoS management and control activities. It has broad application prospects. 


\section{References}

[1] N Simoni, Y Wu, C Yin. QoS Signaling for Service Delivery in NGN/NGS Context [J]. Achi, 2015.

[2] TW Um, J Kim, HW Lee, SH Kim. Dynamic Resource Control Mechanism for Multimedia Overlay Transport in NGN [J]. Multimedia Tools \& Applications, 2013, 65(2):187-199.

[3] Tian Yu. Study on Technical Scheme of IP Network Video Conference System Based on QoS [J]. Henan Science and Technology, 2013(1X):16-16.

[4] Ma Sugang. The Research and Realization of QoS Technology [J]. Electronic Design Engineering, 2015, 23(22).

[5] Jiao Yanping, Li Chang, Li Jianhua. Study on Fusion Technology Based on NGN [J]. Journal of the Academy of Equipment, 2014(1):69-72. 